\title{
Feasibility of Opioid-Free Anesthesia for Cervical Rib Excision: A Case Series and Review of Literature
}

\author{
Souvik Dey ${ }^{1,2(0)}$ Jasvinder Kaur Kohli ${ }^{1,2}$ Rohan Magoon ${ }^{1,2}$ ItiShri ${ }^{1,2}$ Ramesh Chand Kashav ${ }^{1,2}$ \\ ${ }^{1}$ Department of Cardiac Anaesthesia, Atal Bihari Vajpayee Institute of \\ Medical Sciences (ABVIMS), New Delhi, India \\ 2 Department of Cardiac Anaesthesia, Atal Bihari Vajpayee Institute of \\ Medical Sciences (ABVIMS), New Delhi, India \\ Address for correspondence Jasvinder Kaur Kohli, MD, Department of \\ Cardiac Anaesthesia, Atal Bihari Vajpayee Institute of Medical \\ Sciences (ABVIMS) and Dr. Ram Manohar Lohia Hospital, Baba Kharak \\ Singh Marg, New Delhi 110001, India \\ (e-mail: jasviderkohli@gmail.com).
}

J Card Crit Care 2021;5:208-213.

\author{
Abstract \\ Keywords \\ - cervical rib excision \\ - locoregional \\ techniques \\ - multimodal analgesia \\ - opioid-free \\ anesthesia \\ - paravertebral block \\ - superficial cervical \\ plexus block
}

Background Perioperative pain management following cervical rib excision can be compounded in the background of chronic pain disorder caused by the neurovascular compression. The former mandates an enhanced analgesic requirement wherein the perioperative opioid use, in particular, can be associated with a peculiar adverse-effect profile and abuse potential. Appropriate to the context, an opioid-free anesthesia (OFA) protocol can be instrumental in minimizing the incidence of the aforementioned.

Case Series While two patients necessitated OFA owing to opioid contraindication, the formulated protocol was evaluated in another six consecutive patients posted for elective cervical rib excision. A combined paravertebral block and superficial cervical plexus block was employed alongside general anesthesia. Intravenous dexmedetomidine and lignocaine assisted the conduct of OFA, and paracetamol dosing was continued into the postoperative period. Intraoperative rescue analgesia was ensured by a ketofol bolus (1:1 mixture of ketamine and propofol) whereas intravenous diclofenac was used for postoperative rescue analgesia.

Results OFA could be successfully contemplated in all eight patients. A single bolus rescue dose of ketofol had to be administered in two patients intraoperatively and diclofenac had to be administered as postoperative rescue analgesic in two patients. There was no incidence of postoperative nausea/vomiting or any block-related complications. The postoperative stay was uneventful with an acceptable patient satisfaction.

Conclusion The index experience reiterates the fact that a prudent combination of nonopioid multimodal analgesics with case-based locoregional techniques can feature as a successful OFA protocol, albeit mandating future prospective studies in this novel area of clinical interest.
DOI https://doi.org/ $10.1055 / \mathrm{s}-0041-1741492$ ISSN 2457-0206. (c) 2022. Official Publication of The Simulation Society (TSS), accredited by International Society of Cardiovascular Ultrasound (ISCU). All rights reserved.

This is an open access article published by Thieme under the terms of the Creative Commons Attribution-NonDerivative-NonCommercial-License, permitting copying and reproduction so long as the original work is given appropriate credit. Contents may not be used for commercial purposes, or adapted, remixed, transformed or built upon. (https://creativecommons.org/ licenses/by-nc-nd/4.0/)

Thieme Medical and Scientific Publishers Pvt. Ltd., A-12, 2nd Floor, Sector 2, Noida-201301 UP, India 


\section{Introduction}

Cervical rib is the congenital presence of supernumerary rib at C7 vertebra and has an incidence of 0.2 to $0.5 \%$ in general population. ${ }^{1}$ Excision of this extra cervical rib is indicated in the presence of a constellation of symptoms known as thoracic outlet syndrome (TOS) which occurs due to the compression of neurovascular bundle (brachial plexus and subclavian vessels) between this extra rib and first thoracic rib inferiorly and clavicle anteriorly. ${ }^{2}$ Perioperative analgesia constitutes an important component in managing these patients, particularly in the background of chronic pain syndrome resulting from neurovascular compression.

Although opioids have been used as the backbone of perioperative analgesia regime, their use is often complicated by the occurrence of opioid-related adverse drug events (ORADEs). ${ }^{3}$ Respiratory depression, sedation, nausea and vomiting, delayed recovery of bowel movements, and addiction potential classify as the main components of ORADEs. ${ }^{4,5}$ Therefore, an opioid-free anesthesia (OFA) protocol is being adopted in the current era of enhanced recovery after surgery.

The paradigm shift toward OFA is promoted by the introduction of multiple nonopioid analgesics (nonsteroidal anti-inflammatory drugs, N-methyl-D-aspartate antagonists [ketamine], $\alpha-2$ agonists [clonidine, dexmedetomidine], lidocaine, and gabapentin) and safer locoregional techniques. OFA has been contemplated across a wide spectrum of surgical specialties (which includes but not limited to laparoscopic cholecystectomy, bariatric surgery, breast surgery, and cardiac surgery). ${ }^{6-9}$ In the context of rib resection and thoracotomy procedures, paravertebral block (PVB) has been successfully employed with a superior control of perioperative pain over opioids. ${ }^{10,11}$ Motivated by the encouraging existing literature, we pursued an OFA protocol with combined PVB of cervicothoracic (C7-T1) region and superficial cervical plexus block ( $\mathrm{SCPB}$ ) along with general anesthesia in patients with and/or without a known opioid contraindication, coming for an elective cervical rib resection.

\section{Case Series}

The case series included eight consecutive patients aged 18 to 50 years, American Society of Anesthesiologists (ASA) physical status I, scheduled for an elective cervical rib excision (supraclavicular approach) in the time frame from September 2019 to June 2021. While two out of eight patients demonstrated an established opioid contraindication, a written well-informed consent was obtained from the other patients. One of the patients had an allergy to oxycodone group of drugs and the other had a history of past opioid dependency (and had undergone a successful deaddiction). The patients who did not consent for regional anesthesia, preexisting renal and hepatic failure, local anesthetic allergy, coagulopathy, psychiatric illness, chronic opioid use, diabetics, hypertensives, and those on $\beta$-blocker treatment were excluded.
We followed a standard institutional preoperative assessment protocol. In addition, the patients were explained about the simple 11-point Visual Analogue Scale (VAS, 0 for no pain and 10 for worst possible pain) for the assessment of postoperative pain alongside obtaining a written informed consent for a cervicothoracic PVB and SCPB. After shifting the patient to the operating room (OR), standard ASA monitors were attached and an intravenous access was secured.

A perioperative OFA protocol, as outlined in - Fig. 1, was practiced in all the patients. A dexmedetomidine bolus of $1 \mu \mathrm{g} / \mathrm{kg}$ intravenous was given over 15 minutes followed by an infusion at 0.5 to $0.7 \mu \mathrm{g} / \mathrm{kg} / \mathrm{h}$. An ultrasonography-guided PVB was performed in lateral decubitus position using a high-frequency (3-12 MHz) linear transducer (L12-3, Philips Healthcare EPIQ 7 USG machine, Bothell, Washington, United States) after infiltrating the skin with lignocaine. The probe was placed in a parasagittal fashion to the side of operation approximately 2 to $3 \mathrm{~cm}$ lateral to the spinous process of $\mathrm{T} 1$ vertebra. The PVB block was performed with a single injection of $10 \mathrm{~mL} 0.25 \%$ bupivacaine with dexamethasone via 22$\mathrm{G}$ Tuhoy's needle in an in-plane approach from caudalcephalad direction at C7-T1 interspace (-Fig. 2A).

Following the PVB, patients were made supine with neck extended by placing a shoulder roll and head turned opposite to the side of operation. SCPB was performed under ultrasound guidance (after placing the high-frequency linear probe at the posterior border of the sternocleidomastoid muscle with $10 \mathrm{~mL} 0.25 \%$ bupivacaine with dexamethasone via $22-\mathrm{G} 5-\mathrm{cm}$ needle in an in-plane approach from lateralmedial direction) (-Fig. 2B). After confirming paresthesia to the site of skin incision (supraclavicular fossa, -Fig. 2C), induction of general anesthesia with endotracheal intubation was performed with intravenous propofol, midazolam, lignocaine, and atracurium. Anesthesia was maintained by isoflurane at 0.5 to $1 \%$ dial concentration to maintain the bispectral index (BIS) value between 40 and 60 and an atracurium infusion at 7 to $10 \mu \mathrm{g} / \mathrm{kg} / \mathrm{min}$ was used to keep the patient paralyzed.

A decrease in mean arterial pressure (MAP) $>20 \%$ to the baseline value was treated with intravenous fluid infusion and adjusting the dose of isoflurane and dexmedetomidine infusion. An increase of both heart rate (HR) and MAP > 20\% was followed by the administration of rescue analgesic dose of injection ketofol (1:1 mixture of ketamine and propofol). Inhalation isoflurane and atracurium infusion were terminated at the end of operation. All patients received injection paracetamol and ondansetron at the end of the procedure. Injection neostigmine and glycopyrrolate were given after regaining motor power and the patients were extubated in the OR. Thereafter, dexmedetomidine infusion was stopped and patients were shifted to post-anesthesia care unit (PACU). The intraoperative hemodynamic and oxygenation parameters were monitored continuously and documented at predefined time points as enlisted in - Table $\mathbf{1}$. All patients were monitored for hemodynamic and oxygenation parameters and development for any possible block-related complications in the PACU. 


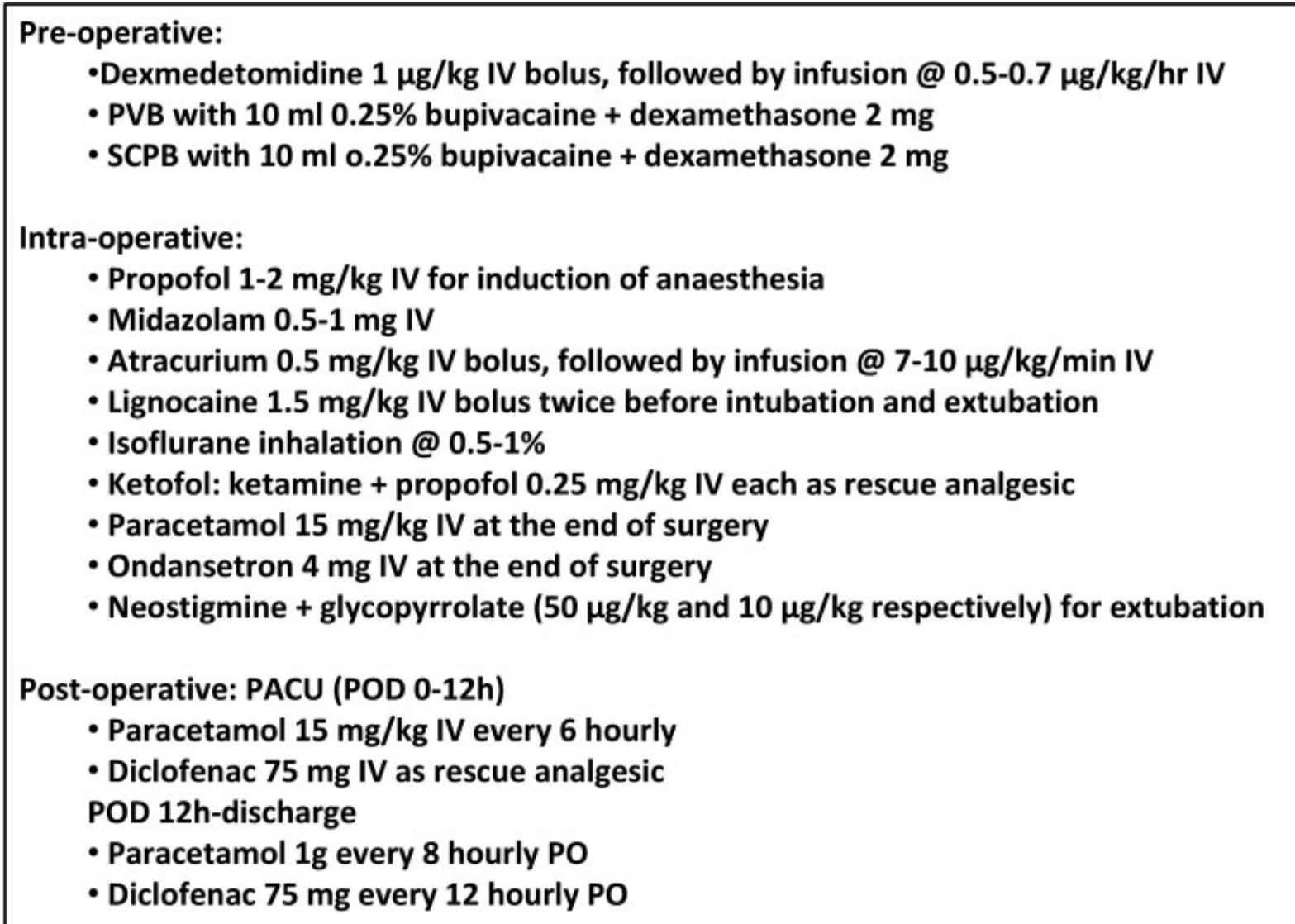

Fig. 1 Opioid-free anesthesia (OFA) protocol employed in the case series with multimodal nonopioid analgesics. IV, intravenous; PO, per oral; POD, postoperative day; PVB, paravertebral block; SCPB, superficial cervical plexus block.
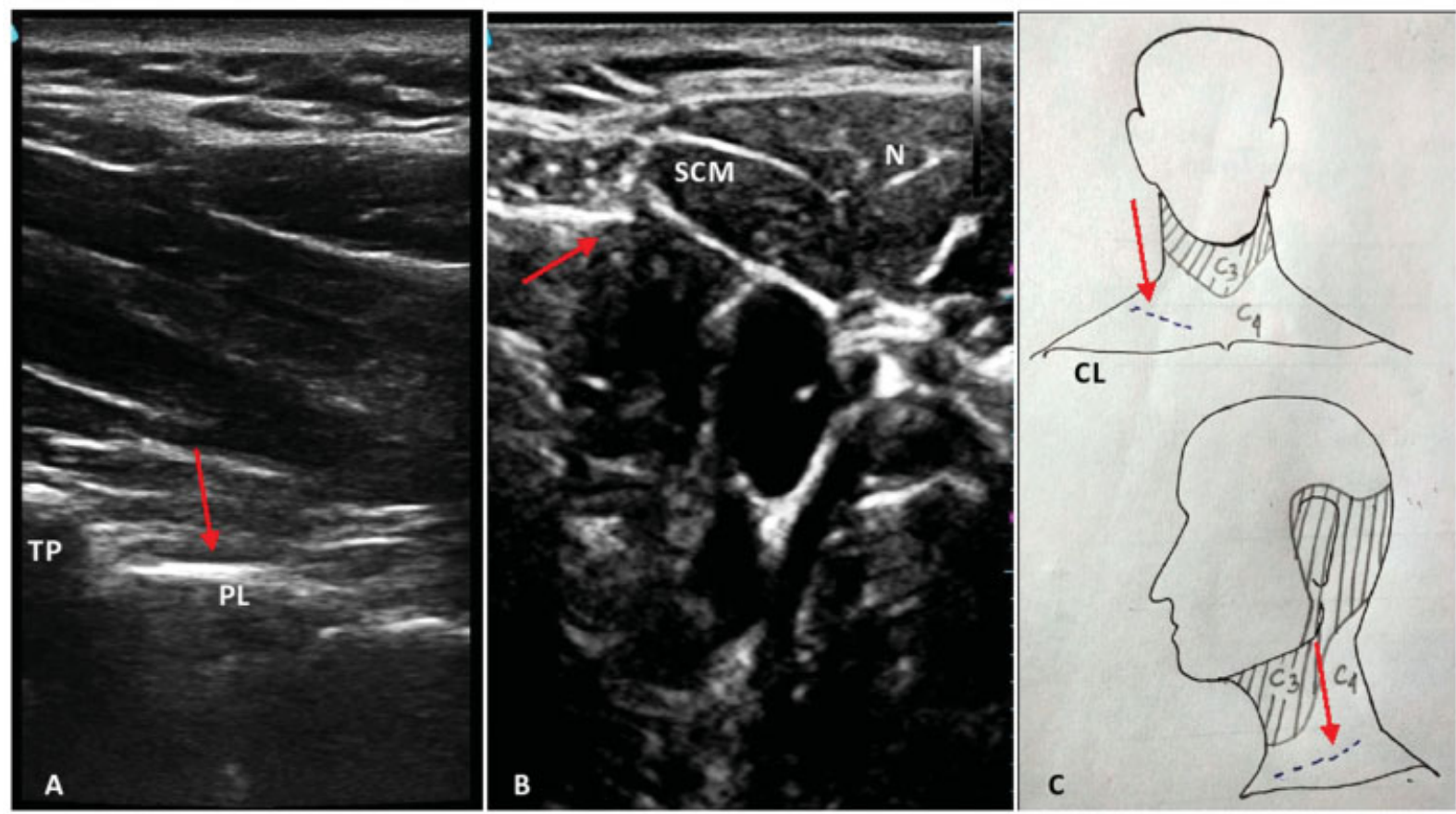

Fig. 2 Sonoanatomy and dermatomal supply of neck with incision line. (A) Sonoanatomy of cervicothoracic (C7-T1) paravertebral space with arrow indicating the space for intended block. (B) Sonoanatomy for superficial cervical plexus block with arrow indicating the plexus. (C) Schematic diagram showing the sensory supply of anterolateral neck with incision line marked by the arrow. CL, clavicle; N, needle; PL, parietal pleura; SCM, sternocleidomastoid; TP, transverse process of C7 vertebra. 
Postoperatively, all patients received injection paracetamol every 6 hourly and VAS and Ramsay Sedation Score ${ }^{12}$ were documented every 2 hourly for the next 12 hours in the PACU. A postoperative VAS $>3$ was considered as the indication of a postoperative rescue analgesic dose of injection diclofenac. Following the attainment of a modified Aldrete Score $\geq 9$, patients were shifted to ward on oral medications.

\section{Results}

OFA could be successfully contemplated in all the patients. During the conduct of OFA, the intraoperative hemodynamics were well maintained in six patients without the requirement of any rescue analgesic. However, an intraoperative increase of both HR and MAP $>20 \%$ were noted in two of our patients without simultaneous increase in BIS value during surgical manipulation. The former was mitigated with the supplementation of nonopioid rescue intraoperative ketofol dosage ( - Table $\mathbf{1}$ ).

Talking of the postoperative period, a total of two out of eight patients complained of pain (VAS $>3$ ) necessitating rescue analgesic of injection diclofenac. While the first patient received rescue diclofenac at the 4th postoperative hour, the other one received at 6 th postoperative hour ( - Table 2 ).

There was no incidence of postoperative nausea/vomiting or any other complications (which included but not limited to nausea and vomiting, bleeding, hematoma formation, hoarseness of voice, difficulty in breathing). The postoperative stay was uneventful with an acceptable patient satisfaction.

\section{Discussion}

Perioperative pain management in cervical rib excision can be challenging owing to its inherent pathology featuring chronic neurovascular compression. Moreover, prolonged use of perioperative opioids additionally raises the potential of ORADEs, opioid overdose, and abuse. Herein, a judiciously formulated OFA regimen containing multiple nonopioid analgesics combined with locoregional techniques might address the abovementioned problem. As an extension of the same, incorporation of tailored regional anesthesia in the multimodal OFA protocol, has been recently demonstrated to be a pivotal contributor to an effective perioperative pain management. ${ }^{13}$

Talking of the procedure-specific regional analgesic techniques, PVB has been described for thoracic surgeries and an effective control of pain following rib fracture. ${ }^{11,14}$ Inclusion of PVB in the anesthetic regimen for excision of first rib causing TOS has been described by Patel et al in their retrospective study with perioperative opioid sparing effect. ${ }^{10}$ Inspired by this parent study, Kalava et al investigated the efficacy of preoperative PVB at T1-T2 interspace for first rib resection via infraclavicular approach for perioperative management of pain. ${ }^{15}$ They found a significant decrease in intraoperative opioid consumption in the PVB group (3.9 \pm 1.8 vs. $12.8 \pm 10.2$ in $\mathrm{mg}$ of morphine equivalent) as compared with the patients who did not receive PVB. ${ }^{15}$

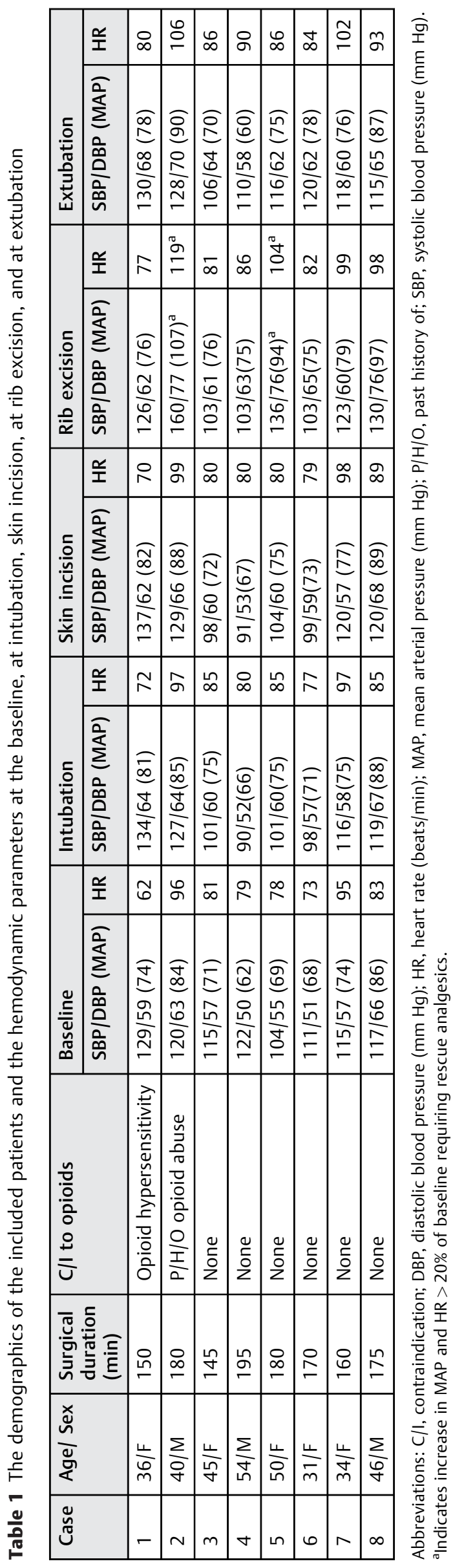


Table 2 Mean postextubation VAS, RSS, number of patients requiring rescue analgesic (diclofenac), and incidence of nausea and vomiting

\begin{tabular}{|l|l|l|l|l|}
\hline & $\begin{array}{l}\text { Mean } \\
\text { VAS }\end{array}$ & $\begin{array}{l}\text { Mean } \\
\text { RSS }\end{array}$ & $\begin{array}{l}\text { No of } \\
\text { patients } \\
\text { requiring } \\
\text { rescue } \\
\text { analgesic } \\
\text { (diclofenac) }\end{array}$ & $\begin{array}{l}\text { Incidence of } \\
\text { nausea } \\
\text { and/or } \\
\text { vomiting } \\
\text { (no of } \\
\text { patients) }\end{array}$ \\
\hline $\begin{array}{l}\text { On } \\
\text { PACU } \\
\text { admission }\end{array}$ & 1.6 & 4.8 & 0 & 0 \\
\hline $2 \mathrm{~h}$ & 1.8 & 4.5 & 0 & 0 \\
\hline $4 \mathrm{~h}$ & 3.2 & 4.1 & 1 & 0 \\
\hline $6 \mathrm{~h}$ & 3.5 & 3.7 & 1 & 0 \\
\hline $8 \mathrm{~h}$ & 2.8 & 3.5 & 0 & 0 \\
\hline $10 \mathrm{~h}$ & 2.6 & 3.1 & 0 & 0 \\
\hline $12 \mathrm{~h}$ & 2.5 & 2.8 & 0 & 0 \\
\hline
\end{tabular}

Abbreviations: PACU, post-anesthesia care unit; RSS, Ramsay Sedation Scale; VAS, Visual Analogue Scale.

In the index case series, the first patient presented with TOS was a known case of opioid hypersensitivity. This condition mandated the adoption of OFA protocol during cervical rib excision. A PVB was chosen over the thoracic epidural anesthesia (TEA) in the view of its better safety profile. ${ }^{16}$ TEA carries the potential risk of permanent neurological injury, hematoma formation, and hypoperfusion of spinal cord. ${ }^{16,17}$ As opposed to TEA, thoracic PVB causes less hypotension and bradycardia and lesser number of pneumothorax. ${ }^{18}$ Motivated by the successful completion of our first two cases of cervical rib excision with PVB and SCPB combined to multiple nonopioid analgesics (dexmedetomidine, lignocaine, paracetamol), we continued the same OFA protocol in the subsequent six patients. We did not observe any block-related complication.

Supraclavicular approach for cervical rib excision requires a sensory blockade of $\mathrm{C} 3$ and $\mathrm{C} 4$ dermatomes. Therefore, a SCPB was performed in addition to C7-T1 PVB for an effective control of pain originating from skin incision and subsequent tissue handling (-Fig. 2C). Additionally, an intraoperative infusion of dexmedetomidine was used as a part of multimodal analgesia. A bolus dose of intravenous lignocaine was used to suppress the laryngoscopic response and extubation response. Maintaining the anesthetic depth at BIS 40 to 60 range with infusion atracurium for muscle paralysis, an increase in MAP with HR $>20 \%$ of baseline would have possibly caused inadequate analgesia in two of our patients. This was addressed by a bolus dose of intravenous ketofol.

Postoperative monitoring of VAS demonstrated an adequate control of pain with intravenous paracetamol except requirement of single dose of diclofenac injection in two of the patients. There was no incidence of postoperative nausea and vomiting. There was no evidence of excessive sedation postoperatively.
To the best of the authors' knowledge, the index case series is the first of its kind demonstrating the feasibility of OFA for cervical rib excision. This becomes particularly relevant in the present era proposing the concept of opioid stewardship. ${ }^{19}$ At the same time, the fact remains that beyond the circumstantial necessitation of OFA, the patient selection can be peculiarly challenging. ${ }^{20}$ In addition, the choice of mechanistically distinct rescue nonopioid analgesics can itself be difficult while contemplating OFA, as in our case. However, in the context of our case series, it is noteworthy that a continuous PVB via catheter insertion could have reduced the requirement of rescue analgesics further.

\section{Conclusion}

Perioperative pain following cervical rib excision can be successfully managed with the assistance of multimodal OFA protocols, as in the index case series. While such OFA protocols captivates attention for their potential of overcoming the ORADEs, larger prospective studies with age- and gender-matched controls ${ }^{21}$ are awaited before adoption in day-to-day clinical practice can be routinely recommended.

\section{Conflict of Interest}

None declared.

\section{References}

1 Guttentag AR, Salwen JK. Keep your eyes on the ribs: the spectrum of normal variants and diseases that involve the ribs. Radiographics 1999;19(05):1125-1142

2 Peek J, Vos CG, Ünlü Ç, van de Pavoordt HDWM, van den Akker PJ, de Vries JPM. Outcome of surgical treatment for thoracic outlet syndrome: systematic review and meta-analysis. Ann Vasc Surg 2017;40:303-326

3 Murthy VH. Ending the opioid epidemic - a call to action. N Engl J Med 2016;375(25):2413-2415

4 Nassif GJ, Miller TE. Evolving the management of acute perioperative pain towards opioid free protocols: a narrative review. Curr Med Res Opin 2019;35(12):2129-2136

5 Magoon R, Choudhury A. Opioid free anesthesia: is it too early to bid adieu? Can J Anaesth 2019;66(10):1268-1269

6 Bakan M, Umutoglu T, Topuz U, et al. Opioid-free total intravenous anesthesia with propofol, dexmedetomidine and lidocaine infusions for laparoscopic cholecystectomy: a prospective, randomized, double-blinded study. Braz J Anesthesiol 2015;65(03): 191-199

7 Tripathy S, Rath S, Agrawal S, et al. Opioid-free anesthesia for breast cancer surgery: an observational study. J Anaesthesiol Clin Pharmacol 2018;34(01):35-40

8 Mulier JP, Wouters R, Dillemans B, Dekock D. A randomized controlled, double- blind trial evaluating the effect of opioidfree versus opioid general anaesthesia on postoperative pain and discomfort measured by the QoR-40. J ClinAnesth Pain Med 2018; 2:1-6

9 Choudhury A, Magoon R, Sahoo S, Sehgal L. Opioid free cardiac surgery: opportunities and obstacles. J Cardiothorac Vasc Anesth 2020;34(02):567-568

10 Patel AN, Finlay KU, Schyra KC, et al. Use of general anesthetic only vs general anesthetic combined with paravertebral block for perioperative pain management after first rib resection. Proc Bayl Univ Med Cent 2002;15(04):374-375 
11 Gessling EA, Miller M. Efficacy of thoracic paravertebral block versus systemic analgesia for postoperative thoracotomy pain: a systematic review protocol. JBI Database Syst Rev Implement Reports 2017;15(01):30-38

12 Ramsay MA, Savege TM, Simpson BR, Goodwin R. Controlled sedation with alphaxalone-alphadolone. BMJ 1974;2(5920):656-659

13 Sultana A, Torres D, Schumann R. Special indications for opioid free anaesthesia and analgesia, patient and procedure related: Including obesity, sleep apnoea, chronic obstructive pulmonary disease, complex regional pain syndromes, opioid addiction and cancer surgery. Best Pract Res Clin Anaesthesiol 2017;31(04):547-560

14 Malekpour M, Hashmi A, Dove J, Torres D, Wild J. Analgesic choice in management of rib fractures: paravertebral block or epidural analgesia? Anesth Analg 2017;124(06):1906-1911

15 Kalava A, Camporesi E, Illig KA, Robards CB. T1, T2 paraverterbal blocks provide opioid sparing in first rib resection for thoracic outlet syndrome. J Clin Anesth 2018;45:4-5
16 Hall Burton DM, Boretsky KR. A comparison of paravertebral nerve block catheters and thoracic epidural catheters for postoperative analgesia following the Nuss procedure for pectus excavatum repair. Paediatr Anaesth 2014;24(05):516-520

17 Meyer MJ, Krane EJ, Goldschneider KR, Klein NJ. Case report: neurological complications associated with epidural analgesia in children: a report of 4 cases of ambiguous etiologies. Anesth Analg 2012;115(06):1365-1370

18 Joshi GP, Bonnet F, Shah R, et al. A systematic review of randomized trials evaluating regional techniques for postthoracotomy analgesia. Anesth Analg 2008;107(03):1026-1040

19 Ardeljan LD, Waldfogel JM, Bicket MC, et al. Current state of opioid stewardship. Am J Health Syst Pharm 2020;77(08):636-643

20 Harkouk H, Fletcher D, Beloeil H. Opioid free anaesthesia: myth or reality? Anaesth Crit Care Pain Med 2019;38(02):111-112

21 Magoon R, Jose J. Safeguarding anaesthesia research from spin. $\mathrm{Br}$ J Anaesth 2020;125(06):e460-e462 